\title{
ESTUDIOS
}

\section{La seguridad y salud en el trabajo en el marco de la responsabilidad social de la empresa}

\author{
María José Montero Simó ${ }^{1}$, Rafael A. Araque Padilla', Juan Miguel Rey \\ Pino $^{2}$
}

Palabras clave: Herramientas de Responsabilidad Social de la Empresa, Seguridad y Salud en el trabajo, Prevención de riesgos, Stakeholders.

Key words: Corporate Social Responsibility Instruments, Occupational Health and Safety (OHS), Risk Prevention, Stakeholders

\section{Introducción}

La sociedad actual está asistiendo a un desarrollo muy intenso de iniciativas que tratan de impulsar y promover lo que se ha venido a llamar Responsabilidad Social de la Empresa (en adelante, RSE), entendiéndose ésta como la integración voluntaria de preocupaciones sociales y medioambientales en la toma de decisiones de las empresas. Ser socialmente responsable significa ir más allá del cumplimiento de la ley, invirtiendo en capital humano y desarrollando relaciones con diferentes actores sociales que se ven afectados por las consecuencias de las decisiones empresariales. No cabe la menor duda de que la búsqueda de un entorno de trabajo seguro y saludable, así como la promoción de una cultura de la prevención de riesgos, son una de las principales responsabilidades sociales de toda empresa, $y$, consecuentemente, una parte integral de la RSE.

En España, al igual que en otros países, nos encontramos con un amplio desarrollo normativo en el ámbito de la prevención de riesgos laborales, pero con un grado de cumplimiento en la empresa muy desigual. Esto último suscita una notable preocupación social. El pasado mes de enero se leía en la prensa española el siguiente texto: (E/ País, editorial 20/01/07):

\footnotetext{
1 Profesores de la Facultad de Ciencias Económicas y Empresariales - ETEA.

2 Universidad de Cádiz.
} 
No hay problema social más persistente en España que el de los accidentes laborales. Los muertos y heridos en el ámbito laboral siguen marcando una vergonzosa diferencia con los mercados laborales europeos. El año pasado murieron 977 trabajadores, apenas 13 menos que en 2005 y se contabilizaron más de 937.000 bajas debido a este tipo de accidentes según el sindicato de Comisiones Obreras (...). A pesar de que esta lacra se conoce y se lamenta desde varios decenios atrás, la pérdida de vidas humanas continúa sin pausa, sin que aparentemente surtan efecto las medidas y regulaciones de seguridad en el trabajo que los Gobiernos de turno escriben en el Boletín Oficial del Estado. Para corregir una epidemia social de esta magnitud es necesario afrontarla como un problema de Estado (...).

Al mismo tiempo, se asiste a un reparto de culpas y acusaciones por parte de los diferentes actores de la prevención. Hasta ahora parece que la estrategia basada en el amplio desarrollo legislativo no ha generado los resultados esperados. La puesta en marcha de estrategias que promuevan una cultura de prevención parece una tarea acuciante.

Trascendiendo las posiciones encontradas en los conflictos relacionados con la seguridad y la salud en el trabajo, ¿es posible resituar el problema de la prevención de accidentes en otro marco más fructífero en ideas y estrategias? ¿Podría el enfoque de la RSE, con sus líneas básicas de voluntariedad, inclusividad de stakeholders y herramientas nuevas de gestión, ser un escenario posible desde donde repensar la estrategia de actuación?

Estas son las cuestiones que pretendemos abordar en el siguiente artículo. Para ello, se ha partido de un estudio cualitativo acerca de la presencia de los asuntos relacionados con la seguridad y salud en el trabajo en las principales herramientas de gestión de la RSE. Tomando como base sus conclusiones se reflexiona sobre el papel que la RSE puede tener en la promoción de una cultura de trabajo saludable. Así, en primer lugar se introducirán algunos aspectos conceptuales, para, a continuación explicar las fuentes y la metodología empleadas en el estudio. Finalmente se expondrán los resultados obtenidos, así como las conclusiones y recomendaciones.

\section{La seguridad y la salud en el trabajo y su entronque con la responsa- bilidad social de la empresa}

El trabajo desempeña una función esencial en las vidas de las personas. Buena parte de nuestra vida transcurre en el lugar de trabajo; a menudo pasamos allí más horas que en otros ámbitos personales y familiares. Sin embargo, a juzgar por las estadísticas, no se puede hablar de que la existencia de entornos laborales seguros y sanos sea una constante. Todos los días del año hay trabajadores en todo el mundo sometidos a multitud de riesgos para la salud, tales como: polvos, gases, ruidos, vibraciones y temperaturas extremadas. Desafortunadamente, algunos empleadores apenas se ocupan de la protección de la salud y seguridad de sus empleados y, de hecho, hay empresarios que ni siquiera saben que tienen la responsabilidad moral, y a menudo jurídica, de proteger a sus trabajadores.

La salud y seguridad en el trabajo (en adelante, SST) constituye una disciplina muy amplia que debe tender a:

- El fomento y mantenimiento del grado más elevado posible de bienestar físico, mental y social de los trabajadores, sea cual fuere su ocupación. 
- La prevención entre los trabajadores de las consecuencias negativas que sus condiciones de trabajo pueden tener en la salud.

- La protección de los trabajadores en su lugar de trabajo frente a los riesgos a que puedan dar lugar los factores negativos para la salud.

- La colocación y el mantenimiento de los trabajadores en un entorno laboral adaptado a sus necesidades físicas o mentales.

- La adaptación de la actividad laboral a los seres humanos.

En otras palabras, la salud y seguridad laborales abarcan el bienestar social, mental y físico de los trabajadores.

Existen pocas dudas en que la preocupación por el bienestar de los trabajadores debería constituir uno de los aspectos más importantes de la responsabilidad social de cualquier empresa. La expresión "responsabilidad social de la empresa», soslayando, de momento, sus diversas interpretaciones, concepciones o contenidos, patentiza, si atendemos a los términos que la integran, las exigencias que la sociedad o las personas manifiestan hacia la empresa como institución social. Estas exigencias se traducen en las expectativas que el conjunto de individuos esperan que sean satisfechas por la empresa dentro de un determinado modelo social, expectativas que comprenden no sólo la creación y distribución de riqueza desde una visión puramente económica, sino, también, la contribución a la resolución de los problemas que la construcción social va planteando.

El concepto de RSE ha ido evolucionando con el transcurso del tiempo ${ }^{3}$, modificándose y enriqueciéndose a partir del interés que ha tenido en los ámbitos académico y empresarial. En el ámbito de la prevención, la RSE puede concebirse como un compromiso voluntario cuyos objetivos y actuaciones deben dirigirse siempre más allá de los niveles mínimos de protección de los trabajadores dispuestos por la legislación nacional y europea. Esto significa velar por que los empleados se beneficien de un nivel de salud y seguridad en el trabajo superior al establecido en la normativa, $y$ tener en cuenta las implicaciones externas tales como la aplicación de criterios de SST en la selección de subcontratistas o en el proceso de comercialización de productos y servicios.

Bien entendida, la RSE puede ser el vehículo que permita acercar la SST a aspectos tan importantes como:

- Recursos humanos.

- Equilibrio entre trabajo y vida familiar.

- Otros derechos fundamentales.

${ }^{3}$ Ver el último Editorial de la Revista de Fomento Socia/(CONSEJo de Redacción, 2006). 
- Cuestiones de medio ambiente.

- Seguridad y salud pública (incluida la seguridad del producto).

- Rentabilidad y productividad.

La RSE puede verse como una oportunidad para integrar los aspectos de SST dentro de un enfoque más amplio; como una oportunidad para abordar las cuestiones de SST más allá -y sobre la base- del cumplimiento de la legislación. También, como se discutirá más adelante, la RSE puede hacer más evidente la relación entre la preocupación social de la empresa y su reputación en el mercado. Aunque en el ámbito de la SST se están desarrollando iniciativas que persiguen presentar de manera positiva las acciones realizadas por la empresa, la RSE puede enseñar cómo capitalizarlas mejor (efecto "aprendizaje"). Ahora bien, ello implica recorrer con prudencia una trayectoria que conduzca a consolidar los logros adquiridos en materia de SST y de RSE, y a la vez que evite el riesgo de saturar la gestión con otra nueva prioridad.

Para poder contrastar realmente el potencial de desarrollo de la SST a través del empuje de la RSE, es necesario analizar la presencia que actualmente tiene en esta área. Así, hemos considerado que el mejor camino para acercarnos a la realidad de la RSE es a través de la revisión de sus instrumentos. A continuación, se exponen las distintas herramientas o instrumentos de gestión de la RSE existentes, clasificándolos, definiéndolos y estableciendo relaciones.

\section{Las herramientas de gestión de la RSE}

Si hay algo que haya constituido una preocupación constante en el ámbito de la RSE, ello ha sido el esfuerzo por convertir una idea en algo que pueda ser gestionado, facilitando así a las empresas las claves de cómo integrarlo en la gestión. Así, hemos asistido al desarrollo de un gran número de instrumentos de gestión, desde principios y códigos de conducta, hasta sistemas de gestión con herramientas tan concretas como indicadores, que facilitarían la concreción y medición. Estos instrumentos pueden categorizarse del siguiente modo (EMPLOYMENT AND SOCIAL AfFAIRS, 2003):

- Principios y Códigos de Conducta: guías que proporcionan pautas generales de comportamiento ampliamente acordadas, pero que adolecen de la falta de mecanismos de auditoría externa. Algunas incluyen un sistema de elaboración de informes propios (por ejemplo, el caso de las empresas adheridas al Pacto Mundial de Naciones Unidas, a la Ethical Trading Initiative $o$ a los Principios Sullivan), mientras que otras están sujetas a vigilancia externa, tanto informal (como ocurre con el seguimiento del código WHO/UNICEF por parte de las ONG), como formal (como es el caso del sistema de Puntos Nacionales de Contacto, que intenta controlar el incumplimiento de los principios establecidos en las guías de la OCDE para las empresas multinacionales).

- Guías para la implantación de sistemas de gestión y certificación: guías auditables para implementar, revisar y obtener certificación externa del cumplimiento con un conjunto de 
estándares. Estos instrumentos permiten que las empresas mejoren sus procesos internos con relación a la responsabilidad social, y proporcionan credibilidad ante diferentes stakeholders mediante sistemas de certificación o verificación.

- Índices de clasificación usados por agencias de inversión socialmente responsable: conjunto de criterios que sirven para identificar y clasificar empresas según su actuación social con vistas a "inversiones socialmente responsables". Generalmente, los fondos de inversión tienen sus propios filtros, de modo que los inversores individuales pueden elegir aquellos productos que se corresponden con sus preocupaciones sociales; aunque también se han desarrollado índices independientes de inversión social por parte de organizaciones como FTSE (Financial Times Stock Exchange) y Dow Jones.

- Esquemas para la elaboración de informes y memorias: se trata de medios (v.gr., AA1000 - GRI) que no se centran en especificar cómo debe actuar socialmente una empresa, sino que aportan un marco para que pueda comunicarse lo que ésta hace con relación a sus stakeholders.

Si bien cada categoría de instrumentos tiene una naturaleza distinta, puede establecerse, no obstante, una relación entre ellas (ver gráfico 1). Esta relación es importante pues nos arroja cierta luz sobre la forma en que la RSE puede ser un factor catalizador de iniciativas de prevención en una organización.

Gráfico I. Instrumentos de RSE

\section{PRINCIPIOS}

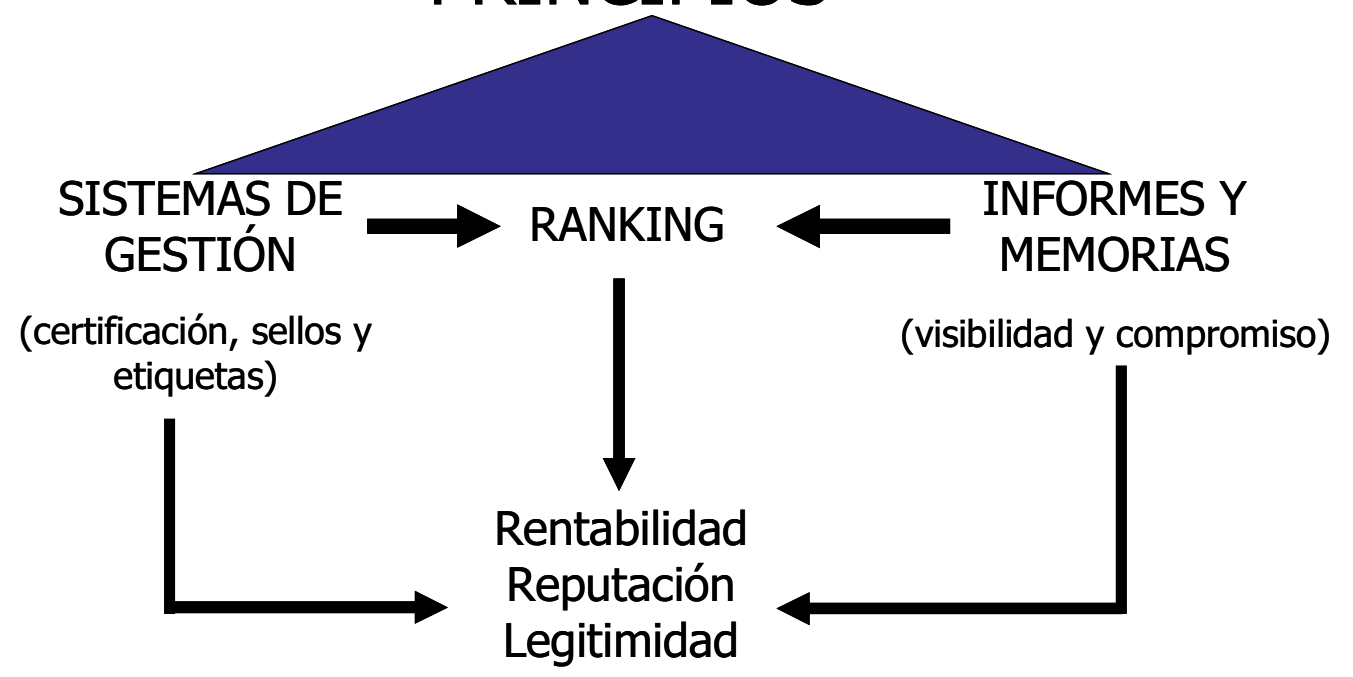

Fuente: Elaboración propia 
Primero, las herramientas basadas en principios y códigos de conducta ampliamente compartidos, aun cuando no pasan de ser un listado de buenas intenciones, constituyen una buena parte de la base axiológica sobre la que se diseñan el resto de herramientas de gestión. Por eso, es importante que las referencias a la SST estén presentes en estas declaraciones de principios, pues eso asegura que van a tener cabida en otros instrumentos de gestión.

Segundo, la implantación de sistemas de gestión y certificación constituye a su vez un criterio de valoración para las agencias que se dedican a la elaboración de índices de clasificación ${ }^{4}$ utilizados para las decisiones de inversión socialmente responsable. Consecuentemente, las empresas que deseen estar bien situadas en esos índices se verán impelidas a incorporar los instrumentos anteriores. Y en la medida en que dichos sistemas de gestión desarrollen cuestiones de SST, también puede esperarse una mejora de su gestión en la empresa.

Tercero, tanto los sistemas de gestión y certificación, como los esquemas para la elaboración de informes y memorias y los índices de clasificación inciden en lo que se ha erigido como uno de los principales atractivos de la RSE -especialmente entre las grandes empresas-: la reputación corporativa. Así, se suele asumir que la gestión de la reputación será de las cuestiones más determinantes para el éxito futuro de las organizaciones. La cuota de reputación que aporta cada una de estas herramientas difiere en su naturaleza. Los sistemas de gestión suscitan interés por su traducción en certificados, sellos o etiquetas que pueden ser exhibidos en el mercado como garantía del comportamiento social. Los informes y memorias aportan visibilidad y compromiso público con la RSE. Y los índices de clasificación añaden el atractivo de la valoración social independiente.

Si suponemos que la empresa busca mejorar su reputación social, puede esperarse una mayor atención a la SST en la medida en que este aspecto se encuentre más desarrollado en los instrumentos anteriores (estrategia de "empuje" o "push"). Pero también desde el ámbito de la SST se puede trabajar para incidir sobre la variable reputación, haciendo ver a la sociedad cómo una empresa no puede legitimarse sin una preocupación y atención adecuadas hacia la creación de un entorno de trabajo seguro y saludable (estrategia de "tirón" o "pull'). A continuación presentamos la metodología seguida para poder analizar la presencia de la SST en el marco de la RSE y así determinar la capacidad de ésta área como estrategia de "empuje".

\section{Metodología}

En este artículo, hemos pretendido estudiar en qué medida las cuestiones de SST están presentes en el ámbito de la RSE para de esta manera poder determinar qué aspectos son los más desarrollados, cuáles quedan más diluidos, y plantear algunas propuestas.

Con este fin hemos optado por una metodología cualitativa basada en el estudio de información secundaria. Concretamente, se ha recurrido a los documentos donde se detalla el contenido de las principales herramientas de gestión de la RSE europeas y/o internacionales. En el gráfico 2 se resume la metodología que se ha seguido.

${ }^{4}$ Ver Camprodon, M.; Sols, J. y Florensa, A. (2006). 


\section{Gráfico 2. Metodología}

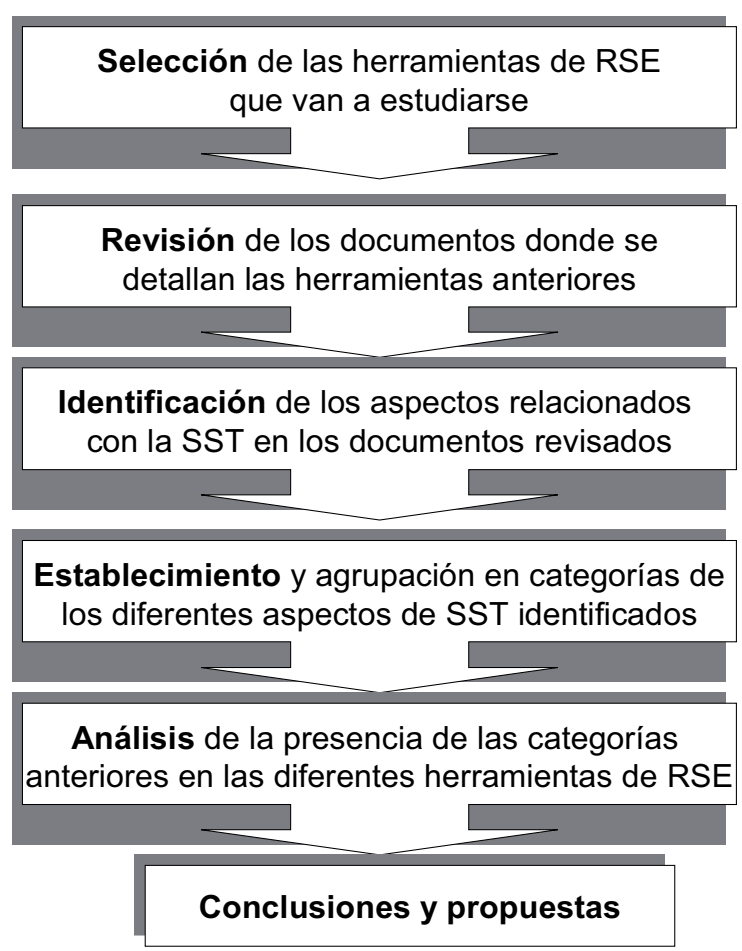

Fuente: Elaboración propia

Se comenzó por seleccionar los instrumentos más representativos a nivel internacional; una vez seleccionadas las herramientas se efectuó una primera revisión exploratoria con el fin de identificar las distintas referencias a la SST presentes en ellas. A partir de aquí, y considerando la totalidad de herramientas de gestión, las distintas referencias se agruparon por categorías y subcategorías con la idea de clarificar y simplificar el análisis. A continuación se volvió sobre los documentos para, en una segunda revisión, organizar la información según categorías y herramientas, obteniendo así los resultados finales del estudio.

\section{I. Fuentes de información}

Para la selección de las herramientas de RSE nos hemos guiado por el Documento de la Unión Europea Mapping instruments for corporate social Responsibility (EMPLOYMENT AND SOCIAL AFFAIRS, 2003). Esta publicación expone un completo análisis estratégico de una serie amplia de herramientas, subrayando sus características más relevantes. En el cuadro 1 se recogen las diferentes herramientas abordadas en ese informe. No se trata por tanto de una revisión exhaustiva, pero sí representativa de los principales referentes internacionales. 
Cuadro I. Instrumentos de RSE analizados

\begin{tabular}{|c|c|c|c|}
\hline PRINCIPIOS Y CÓDIGOS & $\begin{array}{l}\text { SISTEMAS DE GESTIÓN Y } \\
\text { CERTIFICACIÓN }\end{array}$ & $\begin{array}{c}\text { GUÍAS PARA LA } \\
\text { ELABORACIÓN DE MEMORIAS } \\
\text { E INFORMES }\end{array}$ & $\begin{array}{c}\text { INDICES DE RANKINGS DE } \\
\text { EMPRESAS }\end{array}$ \\
\hline $\begin{array}{l}\text { 1. UN Global Compact } \\
\text { (UN GC) } \\
\text { 2. Amnesty International's } \\
\text { Human Rights Guideli- } \\
\text { nes (IA) } \\
\text { 3. Benchmarks for global } \\
\text { corporate responsibility } \\
\text { (ECCR/ICCR) } \\
\text { 4. Ethical Trading Initiative } \\
\text { (ETI) } \\
\text { 5. Global Sullivan Princi- } \\
\text { ples (SULLIVAN) } \\
\text { 6. The OCDE guidelines } \\
\text { for multinational enter- } \\
\text { prises (OCDE) }\end{array}$ & $\begin{array}{l}\text { 7. Social Accountability } \\
8000 \text { (SA 8000) } \\
\text { 8. Sustainability Integra- } \\
\text { ted Guidelines forMa- } \\
\text { nagement (SIGMA) } \\
\text { 9. Forest Stewardship } \\
\text { Council (FSC) } \\
\text { 10. Eco-Managementand } \\
\text { Audit Scheme } \\
\text { 11. EU Eco-Label criteria }\end{array}$ & $\begin{array}{l}\text { 12. Global Reporting Ini- } \\
\text { tiative (GRI) } \\
\text { 13. Accountability } 1000 \\
\text { Series (AA 1000) }\end{array}$ & $\begin{array}{l}\text { 14. Dow Jones Sustaina- } \\
\text { bility Group Index } \\
\text { (DJSGI) } \\
\text { 15. ASPI-Vigeo Group } \\
\text { 16. FTSE4Good Selection } \\
\text { criteria }\end{array}$ \\
\hline
\end{tabular}

Fuente: Elaboración propia

\subsection{Categorización de los elementos de SST}

- Seguridad y Salud en el Trabajo, como principio general.

- Elementos de gestión, incluyendo la referencia de cualquier tipo de estrategia, acción o herramienta propia de la gestión de SST: política documentada, compromiso de mejora continua, procedimientos de evaluación de proveedores, sistemas de detección, evitación o respuesta, implementación y control, auditoria externa, métodos de registro y notificación de accidentes de trabajo y enfermedades profesionales, estadísticas de incidencias, políticas y programas sobre VIH/SIDA, responsabilidad en representante superior, comisiones conjuntas, comunicación externa y sensibilización.

- Derechos de los trabajadores, incluyendo todas las menciones de derechos específicos relacionados con SST: participación, elección de representantes, acceso a servicios de salud, comida saludable, alojamiento digno, agua potable, derecho a detener el trabajo ante la existencia de riesgo percibido, no discriminación por SIDA, prohibición exámenes de salud como condición de empleo, información y formación.

- Legislación y normas: adherencia a códigos y normas, mención a legislaciones nacionales y/o internacionales, y mención expresa a la conveniencia de sobrepasar legislaciones existentes. 
En los siguientes apartados se exponen los resultados obtenidos del análisis realizado, así como las conclusiones y propuestas.

\section{Resultados}

Los resultados se han organizado en el cuadro 2 a partir de las categorías y subcategorías establecidas durante el estudio, identificando las cuestiones relacionadas con la SST que se mencionan con más frecuencia.

\section{- Presencia constante de la SST en los instrumentos de RSE}

Se ha constatado cómo la SST puede considerarse un elemento ineludible de la RSE. Y ello porque:

1. Está presente en todos los instrumentos analizados, con excepción de dos de ellos que abordan aspectos específicos medioambientales (EMAS y Eco-label) y de la norma AA 1000 que, dado su carácter genérico enfocado a procesos relacionales con los stakeholders, no hace mención a otras áreas más concretas.

2. En las declaraciones de principios y códigos de conducta la preocupación por la SST está presente como un principio más, principal, y no como desarrollo de otros.

3. En los principales índices de clasificación para la valoración de empresas con fines de inversión socialmente responsable, la SST es uno de los elementos de valoración, y con un peso relevante.

\section{- Mención indirecta a la SST}

A menudo, las herramientas consideradas no sólo tratan directamente los aspectos relacionados con la SST, sino que también remiten a otras herramientas, así como a otras normas o códigos específicos del sector de la prevención de riesgos, integrándolos como parte de su contenido.

\section{— Escasa aportación de contenidos frente a la legislación en SST}

La mayor parte de la legislación europea está muy desarrollada en cuestiones de SST. Hasta el punto de que su cumplimiento genera no pocos problemas de aplicación y de control o inspección. A la vista de los aspectos mencionados en los instrumentos que se han analizado, podemos decir que no hay nada nuevo que no esté ya reflejado por ley. Lo cual nos lleva a concluir que, en cuanto a contenidos, estos instrumentos no hacen ninguna aportación adicional (si bien en algunos, como es el caso del Forest Stewardship Council, se establece que las actuaciones en este campo deben sobrepasar lo dispuesto en la ley). 
Cuadro 2.Aspectos de SST encontrados en los instrumentos de RSE

\begin{tabular}{|c|c|c|c|c|c|}
\hline & & \multicolumn{4}{|c|}{$\begin{array}{l}\text { PRINCIPIOS Y } \\
\text { CÓDIGOS }\end{array}$} \\
\hline & & UN GC & IA & ECCR/ICCR & ETI \\
\hline \multicolumn{6}{|c|}{ Seguridad y Salud en el lugar de trabajo } \\
\hline \multirow{13}{*}{$\begin{array}{l}\text { Elementos } \\
\text { de gestión }\end{array}$} & Política documentada & & & & \\
\hline & Compromiso mejora continua & & & & \\
\hline & Procedimientos de evaluación de proveedores & & & & \\
\hline & Sistemas de detección, evitación o respuesta & & & & \\
\hline & Implementación y control & & & & \\
\hline & Auditoría externa & & & & \\
\hline & $\begin{array}{l}\text { Métodos de registro y notificación de accidentes } \\
\text { de trabajo y enfermedades profesionales }\end{array}$ & & & & \\
\hline & Estadísticas de incidencias & & & & \\
\hline & Políticas y programas sobre el VIH/SIDA & & & & \\
\hline & Responsabilidad en representante superior & & & & \\
\hline & $\begin{array}{l}\text { Comisiones conjuntas (Dirección y } \\
\text { representantes trabajadores) }\end{array}$ & & & & \\
\hline & Comunicación externa & & & & \\
\hline & Sensibilización & & & & \\
\hline \multirow{9}{*}{$\begin{array}{l}\text { Derechos de los } \\
\text { trabajadores }\end{array}$} & Participación & & & & \\
\hline & Elección representantes & & & & \\
\hline & Acceso servicios salud & & & & \\
\hline & $\begin{array}{l}\text { Comida saludable, alojamiento digno, agua } \\
\text { potable }\end{array}$ & & & & \\
\hline & Derecho a detener el trabajo (riesgo percibido) & & & & \\
\hline & No discriminación por AIDS & & & & \\
\hline & $\begin{array}{l}\text { Prohibición exámenes de salud como } \\
\text { condición de empelo }\end{array}$ & & & & \\
\hline & Información & & & & \\
\hline & Formación & & & & \\
\hline \multirow{3}{*}{$\begin{array}{l}\text { Legislación y } \\
\text { normas }\end{array}$} & Adherencia a Códigos/ Normas & GRI & & WHO/OIT ${ }^{5}$ & \\
\hline & Legislaciones nacionales e internacionales & & & & \\
\hline & Sobrepasar legislación & & & & \\
\hline
\end{tabular}

Fuente: Elaboración propia.

${ }^{5}$ WHO (World Health Organisation);OIT (Organización Internacional del Trabajo). 


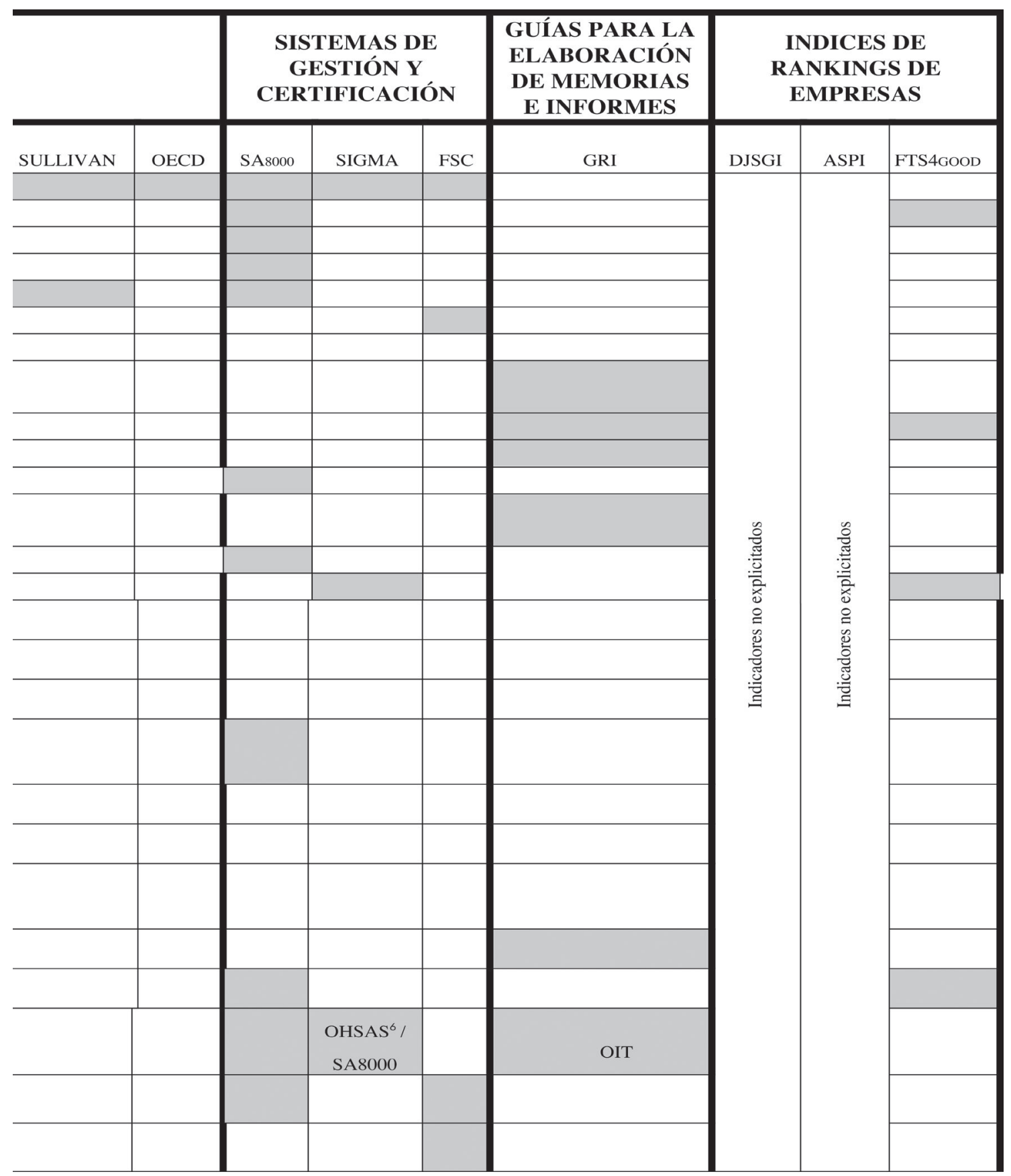

${ }^{6}$ OHSAS (Occupational Health and Safety Assessment Series). 
— Desarrollo desigual entre las herramientas en materia de SST

Conviene destacar el hecho de que dos instrumentos aglutinan la mayor cantidad de menciones explícitas a la SST: Benchmarks for global corporate responsibility (ECCR/ICCR) y Social Accountability 8000 (SA8000). El primero es una iniciativa bastante exhaustiva, con un extenso desarrollo de indicadores de RSE (94 principios éticos, 129 criterios de actuación y 118 indicadores). De ellos, 4 principios, 5 criterios y 7 indicadores corresponden a aspectos de SST. En el caso de la norma SA8000, nos encontramos ante una guía de gestión y certificación centrada en el stakeholder empleado, lo que justifica que haya un mayor número de referencias explícitas a la SST.

\section{— Predominio de elementos de gestión}

Del análisis realizado se desprende una mayor presencia de los elementos de gestión frente a los relativos a derechos de los trabajadores o normas y legislación. Dentro de los criterios de gestión, las principales menciones tienen que ver con: políticas, sistemas de detección y acciones de sensibilización. La preponderancia de estos tres aspectos conecta con el enfoque que desde la Administración Pública pretende dársele a la SST, a saber: la promoción de una cultura de la prevención; una cultura cuyo rasgo más relevante sería la eliminación de riesgos posibles con la intervención de todos los actores (trabajadores y empresarios), de manera planificada y sistematizada, y con base en la educación. Resulta llamativo la escasa mención a los procedimientos de evaluación de proveedores, siendo como es la utilización de subcontratas uno de los puntos más conflictivos en el ámbito de la prevención dada la dificultad de control.

\section{- Escasa referencia a los derechos de los trabajadores}

Se ha observado una escasa referencia a derechos específicos de los trabajadores - a excepción de los Benchmarks for Global Corporate Responsibility (ECCR/ICCR), donde se recogen en un número considerable-. Pensamos que este hecho podría deberse a la exhaustividad con que esos derechos son recogidos en la legislación, lo cual explicaría que muchos instrumentos remitan a la normativa existente. Pero, también, entendemos que haya menciones expresas (probablemente debiera haber más) dado, por un lado, la complejidad de la legislación, y, por otro, el diferente nivel de desarrollo legislativo en los diferentes países. Pensemos que la mayor parte de estos instrumentos han sido creados pensando en la gran empresa multinacional y en sus actuaciones en países con una escasa o nula legislación al respecto. De ahí que no resulte extraño encontrar referencias a derechos muy básicos que en países europeos desarrollados podrían parecer trasnochados.

\section{Conclusiones}

A la vista de los resultados comparativos de las diferentes herramientas, volvemos a retomar la pregunta que se planteaba al principio: ¿̇qué puede aportar en definitiva la RSE -y sus herramientas de gestión- a la SST? A continuación se ofrecen una serie de consideraciones: 
1. Apoyo a la ley. La inclusión de referencias al obligado cumplimiento de legislaciones nacionales e internacionales vigentes relativas a la SST supone un respaldo a la ley. Pero también la RSE puede inspirar nuevas iniciativas de carácter voluntario que ayuden a promover la SST por parte de los actores de la prevención. Esto requeriría de los actores de la prevención (especialmente sindicatos) que se cuestionen críticamente en qué casos las iniciativas voluntarias pueden ser más efectivas que la legislación.

2. Predisposición al diálogo multilateral. Uno de los obstáculos para promover la SST es el clima de tensión que se genera entre los distintos actores de la prevención. Con relación a esto, uno de los aspectos más enriquecedores de la RSE es el concepto y la gestión de relaciones con los stakeholders (ver gráfico 3).

Gráfico 3. Stakeholders en relación con la SST

\section{EMPRESAS}

Empleados (Comité de Seguridad

y Salud; Delegados de Prevención y Técnicos de Prevención)

$$
\text { Directivos }
$$

\footnotetext{
AA.PP.

U.E. (A.E.S.S.T.)

G. Central (INSHT Inspección de

Trabajo y Seguridad Social)

CC.AA.(Organismos

de Prevención)
}

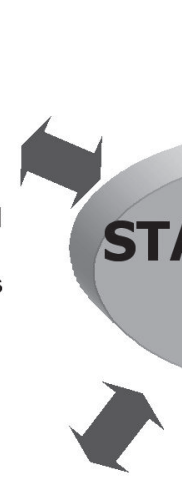

STAKEHOLDERS EN SST

\section{PROVEEDORES}

Servicios de prevención

Subcontratas

Suministradores

Instituciones financieras

\section{INTERMEDIARIOS}

\section{SINDICATOS}

\section{COMUNIDAD}

Consumidores

Org. Civiles

Medios de comunicación

Fuente: Elaboración propia.

Las herramientas de RSE predisponen al diálogo multilateral con los diferentes grupos, lo cual incorpora ventajas como:

- La constitución de partenariados para el diseño de estrategias comunes, estrategias basadas en procesos participativos. Estos procesos suelen tener un mayor calado entre los distintos actores, dada su mayor motivación y eco generado en su difusión. Existen oportunidades para formar partenariados voluntarios e innovadores con stakeholders que normalmente no están implicados en cuestiones de SST, así como para aumentar la concienciación pública y de los medios de comunicación sobre la importancia de la SST. 
- La huída de perspectivas unilaterales evitando la confrontación. El diálogo multistakeholder predispone hacia una mayor empatía entre actores, buscando más los puntos comunes que los divergentes en la búsqueda de la consecución del objetivo que todos persiguen.

- Una visión de la SST desde un punto de vista más amplio que el estrictamente económico. Este diálogo permite plantear la existencia de otro tipo de intereses que están en juego, que, si sólo se midieran según su valor en términos económicos, probablemente no podrían llegar a cuestionarse nunca, siendo en muchas ocasiones más importantes que ningún otro tipo de interés.

- La participación de stakeholders no directamente relacionados con el ámbito de la prevención, como intermediarios de apoyo para las estrategias que se definan. En múltiples ocasiones se puede ser mucho más efectivo, para alcanzar un determinado público, a través de terceras personas que actúan como prescriptores del mensaje que se quiere transmitir. Tales intermediarios tienen un poder de influencia mucho mayor sobre el receptor del mensaje, ya sea por la existencia de autoridad moral, simbólica o por su experiencia.

3. Procesos más claros y sistemáticos para comunicar y medir resultados e impactos. La claridad y concreción expositiva de los elementos de SST en los instrumentos analizados, así como la existencia de indicaciones para su puesta en práctica facilitan enormemente la aplicabilidad de la ley.

4. Integración en la gestión normal de la empresa. El enfoque de la RSE puede ayudar a integrar la SST en la gestión empresarial como un elemento transversal, y no como un área marginal o paralela a la actividad ordinaria, contribuyendo así a poner de relieve un aspecto a menudo olvidado o minimizado en la empresa.

5. Atención a procesos, no solo a resultados. El hecho de que la RSE no sólo preste atención a la consecución de determinados resultados, sino que se oriente también al desarrollo de procesos que aseguren un comportamiento socialmente responsable puede significar una mejora para la planificación y gestión de la SST. Es decir, la preocupación por mejorar los procesos de gestión empresarial con una filosofía de RSE puede producir un efecto de arrastre en la gestión de aspectos sociales como los que venimos analizando.

6. El atractivo de la valoración social y económica. La RSE se ha constituido como un factor de empuje debido a distintos factores como la búsqueda de la reputación, de legitimación o de diferenciación, en un mercado donde los atributos éticos de los productos son cada vez más valorados por un segmento creciente de consumidores. La inserción en la estrategia de responsabilidad social de intereses relacionados con la prevención de riesgos puede incrementar ese atractivo de mercado.

Como se ha destacado anteriormente, no todos los instrumentos desarrollan con la misma intensidad las cuestiones de SST, de ahí que dependiendo de la aceptabilidad social que cobre cada uno de estos instrumentos tenderán a desarrollarse más unas cuestiones que otras. 
Por último, nos gustaría añadir que el enfoque que se percibe en todos los instrumentos analizados hacia la SST está en consonancia con la orientación que generalmente se tiene de ella desde la Administración pública, basada en la minimización de conductas de riesgo y en un modelo de promoción basado en el miedo a las consecuencias por incumplimientos ${ }^{7}$. No obstante, existen otras corrientes que apuestan más por la promoción de conductas seguras (J. M. GUTIÉRREZ, 2005a, 2005b), lo que representa una filosofía diferente de gestión, más motivadora y estimulante para el trabajador. La inmensa mayoría de los actuales sistemas de gestión de prevención se establecen en función de la evitación de accidentes. Su misión se basa, por tanto, en un objetivo de negación que presupone en ellos inevitablemente un enfoque reactivo. La visión alternativa propuesta se basa en el paradigma cognitivo-conductual que busca de forma positiva las conductas seguras desde un enfoque proactivo. A través de la "ingeniería de personas" se centran los esfuerzos en la promoción de las mejores conductas individuales y de grupo. Todo ello sin olvidar la comprobación de que, previamente, han sido cubiertas sobradamente las necesidades técnicas de seguridad. Se procede a un análisis y evaluación pormenorizados de las conductas inseguras para intentar comprender su génesis y los beneficios que cada sujeto recibe por saltarse la norma establecida. Así, se persigue conseguir modificar las condiciones que favorecen la ocurrencia de las conductas inseguras $y$, por tanto, aumentar la frecuencia de las conductas seguras. Se fomenta que la cultura de la empresa y la "presión" de los compañeros promueva en los trabajadores una única manera da proceder: la correcta. Trabajar con conductas seguras viene arrojando resultados excelentes en el descenso de la accidentalidad, y además, asegura que las actitudes de los trabajadores se mantengan alineadas de forma homogénea con los valores de Seguridad, Salud y Calidad de vida laboral propuestos por la dirección de la empresa.

Si finalmente se extendiera este enfoque positivo de la SST, sería en todo punto necesario modificar y crear nuevos indicadores para incorporarlos en los instrumentos de RSE. Sin duda, lo anterior sería difícil de conseguir sin una presencia mayor de los problemas relacionados con la SST en los foros multistakeholders sobre la RSE. Por ello, se antoja necesario estrechar los vínculos entre ambas áreas, por parte de los profesionales de la SST y de la RSE, concibiendo la SST como una parte integrante, ineludible de la RSE. Será entonces cuando el desarrollo actual de la Responsabilidad Social pueda servir realmente de estrategia de empuje para la labor de Prevención en Riesgos Laborales.

\footnotetext{
7 Recientemente, se ha conocido por la prensa española que el BOE identificará a las empresas sancionadas por riesgos laborales, a partir de un decreto ley aprobado por el Consejo de Ministros. La identidad de las empresas sancionadas quedaba hasta ahora preservada de la luz pública. Sólo la Inspección de Trabajo conocía quiénes eran, al investigarlas e imponerles la correspondiente sanción. La publicación persigue divulgar las malas prácticas con el objeto de frenarlas.
} 


\section{Bibliografía}

Aragón, J; RoCHA, F. y CRUCES, J. (2005), La dimensión laboral de la RSE en España: un análisis de casos, Fundación $1^{\circ}$ de Mayo.

Araque, R. A. (2002): “Una aportación al debate sobre el Libro Verde de la responsabilidad social de la empresa", Revista de Fomento Social, n 228, vol. 57, pp. 595-628.

Araque, R. A. y Montero, M. J. (2006), La responsabilidad social de la empresa a debate, Barcelona, Icaria Editorial.

Argandoña, A. (1998), "The Stakeholder Theory and the Common Good", Journal of Business Ethics, 17, pp. 1093-1102.

Bestratén Bellovi, M. and Pujol Senovilla, L. (2004), "Responsabilidad social y condiciones de trabajo", Revista Prevención, trabajo y salud n 31, pp. 25-40.

BoIx, P.; GIL, J.M. AND RODRIGO, F. (2006), Prioridades estratégicas en salud laboral desde el punto de vista de los profesionales, Observatorio de Salud Laboral.

Carroll, A. B. (1999), "Corporate Social Responsibility. Evolution of a Definitional Construct", Business and Society, vol. 38, n 3, pp. 268-295.

Camacho laraña, I. (2004), "La responsabilidad social de la empresa: un proceso abierto", Revista de Fomento Social, n 233, vol. 59, pp. 75-98.

Camprodon, M.; Sols, J. y Florensa, A. (2006), "Las Agencias de evaluación de la Responsabilidad Social Corporativa: El estudio de un caso", Revista de Fomento Social, n² 243, vol. 61, pp. 393-422.

COMISIÓN DE LAS COMUNIDADES EUROPEAS (2001), Libro Verde. Fomentar un marco europeo para la responsabilidad social de las empresas [en línea], Bruselas, Com (2001) 366 Final, [Consulta: 23 octubre 2001].

Comisión de las Comunidades Europeas (2002), Comunicación de la Comisión relativa a la responsabilidad social de las empresas: una contribución empresarial al desarrollo sostenible [en línea], Bruselas, Com (2002) 347 Final, [Consulta: 2 noviembre 2002].

CONSEJO de RedACCIÓN (2006), "La responsabilidad social de la empresa: ¿̇el coste de tener conciencia?", Revista de Fomento Social, n 244, vol. 61, pp. 499-517.

Cruañas Acosta, A. (2005), "¿’Podemos competir siendo Socialmente Responsables?", Gestión Práctica de Riesgos Laborales, $\mathrm{n}^{\circ} 15, \mathrm{pp} .47-50$.

EMPLOYMENT AND SOCIAL AfFAIRS (2003), Mapping instruments for corporate social Responsibility, Bélgica, Official Publications of the European Communities. 
European Agency for Safety and Health at Work (2002), "Corporate social responsibility and health at work", Forum n 3.

European Agency for Safety and Health at Work (2005), "Eficacia de los incentivos económicos para mejorar la seguridad y salud en el trabajo", Forum n ${ }^{\circ} 14$.

European Agency for Safety and Health at Work (2004), Corporate social responsibility and safety and health at work (Research), Bélgica, Official Publications of the European Communities.

FundACIÓN EUROPEA PARA LA MEJORA DE LAS CONDICIONES DE TRABAJO (2003), La responsabilidad social de las empresas y las condiciones de trabajo, Dublín, Fundación Europea para la mejora de las condiciones de trabajo.

GutiérRez, J. M. (2005a), "Prevenir en positivo (I)", Gestión Práctica de Riesgos laborales n² 21, pp. 10-11.

GutiérRez, J. M. (2005b), "Prevenir en positivo (III)", Gestión Práctica de Riesgos laborales n 22, pp. 10-11.

JONES, M. T. (1999), "The Institutional Determinants of Social Responsibility", Journal of Business Ethics, 20, pp. 163-179.

Ribelles Villalba, A.; CapdeVila García, l.; Martínez Oyarzabal, S.; Sánchez lloris, R.; Villar Mira, S. Y ZAPATERO JORDÁ, R. (2006), "ARSISAT: Una herramienta par ala autoevaluación en RSC interna respecto a las salud de los trabajadores", Gestión Práctica de Riesgos Laborales, n 23, pp. 29-31.

SÁenz Blanco, M.T. (2006), "Arcelor: Cómo integrar la PRL en la política de desarrollo sostenible", Gestión Práctica de Riesgos laborales n² 23, pp. 14-18.

SWOden, P. AND SINHA SUNIL (2005), Promoting health and safety as a key goal of the Corporate Social Responsibility agenda, United Kingdom, Health \& safety Executive Books.

Wheeler, D. y SiLlanpä̈̈, M. (1997), The stakeholder corporation, Londres, Pitman Publishing.

\section{Páginas Web (Herramientas de RSE)}

Institute of social and ethical Accountability www.accountability.org.uk

Amnisty International www.amnesty.org.uk/business/pubs/hrgc.shtml

Vigeo-Corporte social Responsibility Rating www.arese-sa.com

Dow Jones Sustainability Indexes www.sustainability-index.com 
The Global Principles Steering Group www.web.net/-tccr/benchmarks

European Union www.europa.eu.int/comm./environment/emas

Ethical Trade Initiative www.ethicaltrade.org

European Union www.europa.eu.int/comm./environment/ecolabel

Forest Stewardship Council www.fscoax.org

FTSE The Independent Global Index Company www.ftse4good.com

Global Reporting Initiative www.globalreporting.org

Organization for Economic Cooperation and Development Guidelines for Multinational Enterprises www.oecd.org/daf/investment/guidelines

Social Accountability International www.cepaa.org

The Sigma Project www.projectsigma.com

Global Sullivan Principles of Social Responsibility www.globalsullivanprinciples.org

United Nations Global Compact www.unglobalcompact.org 